\title{
In-Situ Conjugation - Tailored Nanoparticle-Conjugates by Laser Ablation in Liquids
}

\author{
Svea Petersen, Jurij Jakobi, Angelika Hörtinger, Stephan Barcikowski \\ Laser Zentrum Hannover e.V.; Hollerithallee 8, 30419 Hannover \\ s.petersen@lzh.de
}

\begin{abstract}
The generation and characterisation of nanoparticulate carrier systems is important for drug delivery, biosensing and in vivo or in vitro diagnostics. Conventionally, nanoparticle generation is based on chemical synthesis methods requiring time intensive reaction and additive design for each material. Successive purification and surface functionalisation is often required after the nanoparticle generation to achieve pure nanoparticle-conjugates.

Ultra short pulsed laser ablation in liquids has evolved as a powerful tool for nanoparticle generation showing advantages in the nearly unlimited material variety and purity. We perform the ablation in solutions containing bioactive reagents, realizing an in-situ conjugation of nanoparticles. This novel method enables the generation of diverse nanoparticle conjugates, demonstrated at the example of dodecanthiol stabilized gold nanoparticles in hexane. A first bioconjugation with oligonucleotides was carried out additionally. We studied the influence on productivity of nanoparticle generation and evaluated the integrity of biomolecules after laser ablation. A quenching of nanoparticle growth and narrowing of the surface plasmon resonance, which both increases in media with higher conjugative agent concentrations is observed. Since laser generated gold nanoparticles show electron accepting properties due to a partial oxidation, the presented in-situ conjugations can be exploited to other application, e.g. the coupling to polymeric matrices, as far as the conjunctive molecules bear electron donor moieties.
\end{abstract}

DOI: $10.2961 /$ jlmn.2009.01.0014

\section{Introduction}

Nanoparticle-conjugates show widespread applications in medicine as they are able to induce a certain function depending on nature, structure and surface activity (e.g. hyperthermia [1], imaging [2], gene regulation [3] or antisensing). Especially, gold nanoparticles are of increasing interest for cell imaging [4] and sensing due to their inert properties concerning cytotoxicity and their higher magnitude of light scattering compared to molecular fluorophores [5].

Any process designed to generate such nanoparticulate systems needs to consider the application prospects of generated nanoparticles and the time required for generation. Conventionally, nanoparticle generation is well established by chemical synthesis methods. The required functions for targeting (e.g. antibodies), enhanced cellular uptake (e.g. peptides) etc. can be achieved in a successive surface functionalisation after the nanoparticle generation (Figure 1). However, this requires time intensive adaptation for each material and their biomedical application might be restricted due to the risk of impurities, caused by the use of additives and chemical precursors[ 6].

The present paper introduces a novel preparation method, enabling a single step generation of pure nanoparticleconjugates by laser ablation in liquids. This method consists in the laser ablation of a target in liquid media containing conjunctive agents. The novel approach takes advantage of the purity of nanoparticles, generated by laser ablation of solid targets in liquids without the use of chemical precursors [7, 8, 9]. Laser generated gold nanoparticles show electron accepting properties due to a partial oxidation. This property is a prerequisite for in-situ functionalisation, as it implies a tendency of dispersed additives bearing electron donor moieties as $\mathrm{NH}_{2}, \mathrm{COOH}$, $\mathrm{SH}, \mathrm{S}-\mathrm{S}$, etc. to coordinate to the nanoparticle's surface
[10]. The formation of strong dative bonds between thiols and gold surfaces in general has been extensively studied by Brust et al. [11], while in the last years observations concerning the stabilization of a gold colloidal solution with alkylthiols during laser ablation are discussed [12,13]. Compagnini et al. could reveal the formation of Au-S bonds during laser ablation by IR-spectroscopy.

In this paper we therefore chose dodecanthiol (DDT) and thiolated oligonucleotides as model substances for stabilization in hexane and bioconjugation in aqueous solution respectively, so that we added DDT or single stranded oligonucleotides ( $\mathrm{ssO}$ ) to media prior to ablating a gold foil. The novel approach is discussed with regard to nanoparticle productivity and integrity of ssO after laser ablation. The influence of the conjugative agent concentration on the size distribution and optical properties of conjugates is determined.

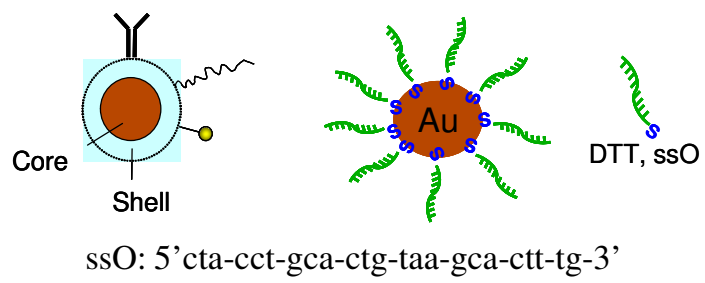

Figure 1 Scheme of a bioconjugate (left) and the ssO or DDT conjugated gold nanoparticle (right)

\section{Experimentals}

\subsection{Materials}

The oligonucleotides were purchased from Biospring GmbH (D-60386 Frankfurt am Main) (ssO: 5'cta-cct-gcactg-taa-gca-ctt-tg-3', Mod. 5':C6-Disulfide) and the gold 
foil (thickness: $0.1 \mu \mathrm{m}$, purity $>99.99 \%$ ) from Goodfellow GmbH (D-61213 Bad Nauheim). Oligonucleotides omitted the treatment with dithiothreitol prior to conjugation, which is usually carried out to ensure that the thiol modified oligonucleotide is in a mono-thiol form rather than disulphide when applied to nanoparticles. Dougan et al. have observed that the conjugation process is even improved when using alkyl-thiolated oligonucleotides as there is no trade off in conjugate stability or surface coverage as a result of omitting reduction with dithiothreitol [14].
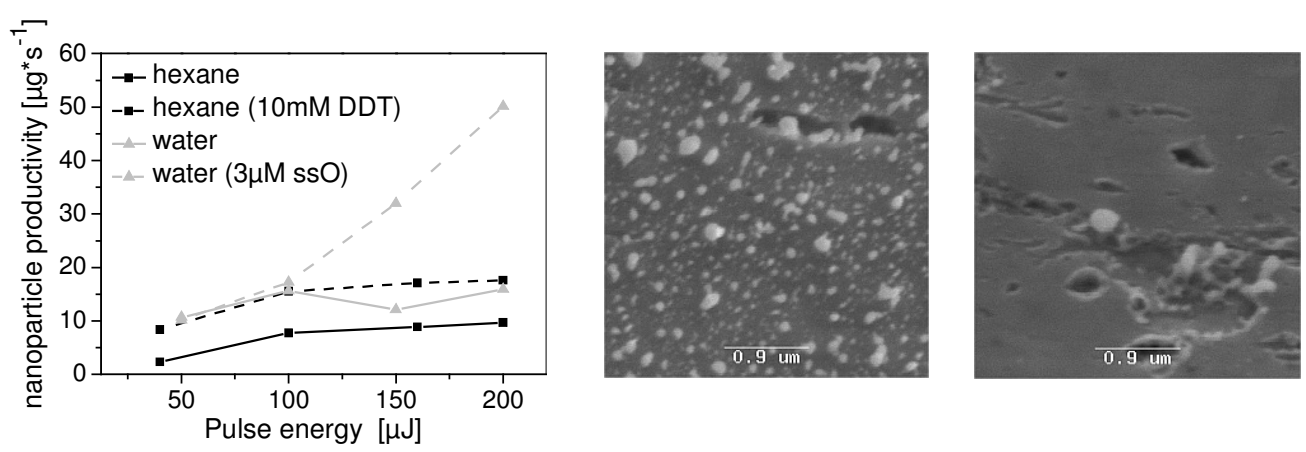

Figure 2 (left to right) Dependence of nanoparticle productivity after 15 minutes of ablation on the pulse energy in different ablation media and SEM micrographs of redeposits on gold target after ablation at $200 \mu \mathrm{J}$ in aqueous media without and with ssO

\subsection{Nanoparticle generation method}

Laser generation of gold nanoparticles was carried out using a femtosecond laser system (Spitfire Pro, SpectraPhysics) delivering $120 \mathrm{fs}$ laser pulses at a wavelength of $800 \mathrm{~nm}$ (maximum energy: $400 \mu \mathrm{J}$ per pulse, beam diameter: $4 \mathrm{~mm}$ ). If not indicated differently, the pulse energy was fixed to $100 \mu \mathrm{J}$ at a repetition rate of $5 \mathrm{kHz}$ resulting in a beam waist of $270 \mu \mathrm{m}$ at the target. The principle set-up is described as follows: the laser beam is focussed on a $5 \times 5 \mathrm{~mm}$ gold foil, placed on the bottom of a petri dish filled with $500 \mu \mathrm{L}$ of a $3 \mu \mathrm{M}$ aqueous solution of ssO $\left(\mathrm{H}_{2} \mathrm{O}:>18 \mathrm{M} \Omega \mathrm{cm}\right)$ or $10 \mathrm{mM}$ DDT in hexane. The depth of the layer above the target was $1 \mathrm{~cm}$. The plate was placed on an axis-system, that moved at a constant speed of $1 \mathrm{~mm}^{*} \mathrm{~s}^{-1}$ in a spiral with outer radius of $1 \mathrm{~mm}$ and inner radius of $0.4 \mathrm{~mm}$.

\subsection{Characterisation methods}

UV/VIS Spectra of the colloidal solutions were recorded in the spectral region of 230-800 nm (Shimadzu 1650).

Size measurements were performed by Dynamic Light Scattering (DLS) with the Zetasizer ZS (Malvern). Typically $200 \mu \mathrm{L}$ of the colloid were added to $800 \mu \mathrm{L}$ water and the resulting dilution was injected into the cuvette. Three consecutive measurements are carried out.

For the determination of the nanoparticle productivity the gold foils were weight three times prior and after ablation on a Sartorius balance (accuracy: $1 \mu \mathrm{g}$ ).

Integrity of ssO was determined by performing polyacrylamide gel electrophoresis of media after ablation at defined process parameters. The ablation media after nanoparticle generation were treated with a $100 \mathrm{mM}$ aqueous dithiothreitol-solution. Dithiothreitol was used to rapidly displace the surface bound ssO via an exchange reaction and induce nanoparticle aggregation as consequence. This method guarantees that conjugated and unconjugated $\mathrm{ssO}$ are analysed by gel electrophoresis. The references, containing the same concentration of $\mathrm{ssO}$, omitted the laser irradiation and were treated equally before gel electrophoresis and run with the samples on each gel. To visualize the bands on the gel after gel electrophoresis, gels were stained and developed using FastSilver ${ }^{\mathrm{TM}}$ (G Biosciences, USA). Scans of resulting stained gels were analyzed with Image J. The intensity of the bands (I(x)) was compared to the references $(\mathrm{I}(0))$ in order to deduce the amount of remaining intact $\mathrm{ssO}$.

\section{Results and Discussion}

\subsection{Influence on nanoparticle productivity and biomolecule degradation}

Generally, the ablation efficiency and nanoparticle yield increases with increasing pulse energy at constant focal position. But at the same time, the heat impact to material and the risk of molecule degradation due to heating also increases. But for in-situ bioconjugating nanoparticles, a negligible degradation of biomolecules after interaction with the laser beam and a sufficient productivity of nanoparticle generation have to be obtained.

We first studied the nanoparticle productivity at differrent pulse energies and compared results to gold nanoparticles generated in pure water and hexane with and without DDT. As expected, the productivity increased with increasing pulse energies in all media. But results evidence significant differences in productivity in the four ablation media. While the productivity in pure hexane is less than $10 \mu \mathrm{g}^{*} \mathrm{~min}^{-1}$, already a productivity of $15 \mu \mathrm{g} * \mathrm{~min}^{-1}$ can be achieved in water and hexane containing DDT. A further increase in the yield is detected at pulse energies of $150 \mu \mathrm{J}$ and $200 \mu \mathrm{J}$ in the aqueous biomolecule solution (Figure 2). Assuming that the difference in the optical properties between pure and stabilizing agents containing solutions is negligible, the ablation process itself should not be affected by the ablation media. Therefore the same amount of gold is supposed to be ablated in both cases. Consequently without ssO or DDT in solution, we assume that particles redeposit on the target's surface after ablation, when reaching the stabilization limit. This assumption is confirmed by SEM micrographs of a gold target after ablation at $200 \mu \mathrm{J}$ in water and aqueous ssO solution (Figure 2). 
While laser generated nanoparticles are electrostabilized in water due to a partial oxidation by present oxygene, they agglomerate in unpolar solvents as hexane if not sterically stabilized by e.g. DDT. In an aqueous ssO solution, both stabilization modes are active, resulting in electrosterical stabilized nanoparticles. This explains why we generally observe increased productivities in water compared to hexane. In order to further define process parameters for the in-situ bioconjugation, we studied the degree of ssO degradation at $100 \mu \mathrm{J}$ and $200 \mu \mathrm{J}$ after different laser irradiation times by gel electrophoresis (Figure 3). At optimal parameters, negligible degradation should be guaranteed.

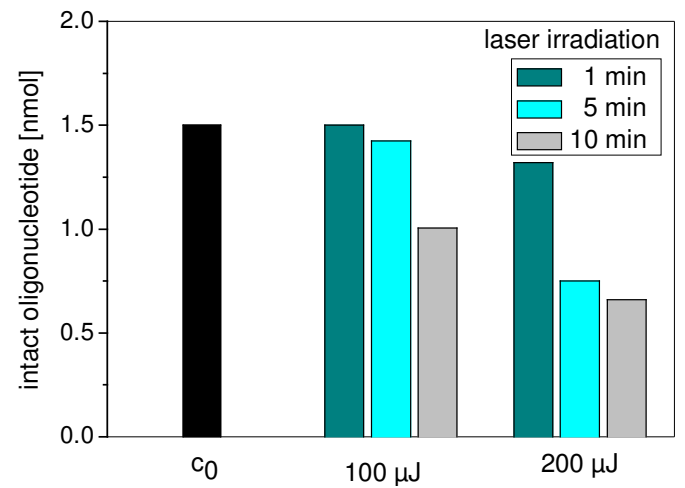

Figure 3 Amount of intact ssO after nanoparticle generation at 100 and $200 \mu \mathrm{J}$ after different irradiation times

We observed a decrease in the amount of intact $\mathrm{ssO}$ with increasing applied pulse energy and increasing time. The time dependence might be due to nanoparticles interfering with any laser pulse, following their generation, prior to ablation of the target. Depending on the pulse peak power, the laser-nanoparticle interference provokes the formation of nanoplasma around gold nanoparticles. This can trigger the degradation of biomolecules, as reported by Takeda et al. at the example of adsorbed lysozymes onto the surface of gold nanoparticles [15].

Generating nanoparticles at $100 \mu \mathrm{J}$ for $53 \mathrm{~s}, 100 \%$ integrity is detected. As nanoparticle-bioconjugate productivity is $20 \mu \mathrm{g}^{*} \mathrm{~min}^{-1}$, this parameter was chosen for further in-situ conjugation experiments.

\subsection{Influence on nanoparticle size distribution and optical properties}

The size distribution and the optical properties are also significantly influenced by conjugation. The hydrodynamic diameter (indicated by the $\mathrm{d}_{\mathrm{h} 50}{ }^{16}$ ) showed a reciprocal dependency on the concentration of ssO (Figure 4). This is in well accordance with data obtained in a previous study for conjugation with DDT in hexane 17. It seems that a critical concentration of $\mathrm{ssO}$ is required to achieve a significant growth quenching.

As the concentration falls below $0.5 \mu \mathrm{M}$, agglomerates are detected. At higher concentrations no further significant changes were observed in the size distribution.

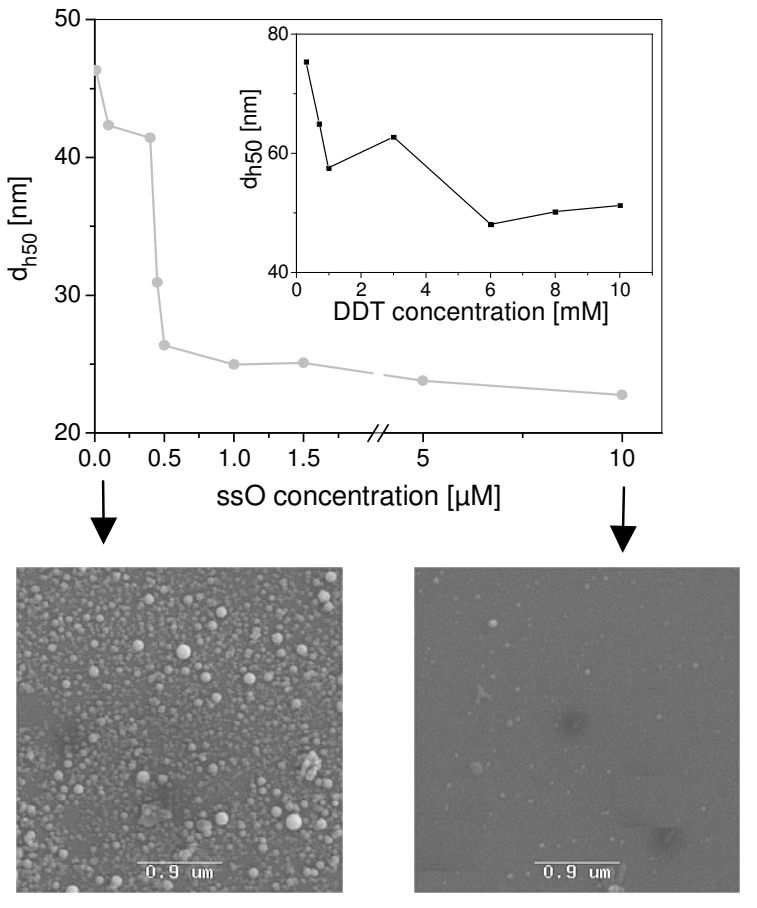

Figure 4 Influence of ssO and DDT concentration on the hydrodynamic diameter determined by DLS and SEM

The critical concentration of $0.5 \mu \mathrm{M}$ could be confirmed by UV-VIS spectroscopy. At lower concentrations, the surface plasmon resonance band at $520 \mathrm{~nm}$, characteristic for gold colloids, is significantly broadened, indicating the presence of (asymmetric) agglomerates in solution. In the spectra of bioconjugates, one observes a second band at $260 \mathrm{~nm}$, increasing with increasing ssO concentration. This band results from electronical shifts in purines and pyrimidines contained in the oligonucleotide. At LPM 2007, we showed a similar change in the optical properties of a gold colloid when increasing the DDT concentration in hexane as ablation medium [18]. In agreement to these findings, we report quenching of nanoparticle growth and narrowing of the surface plasmon resonance as evidence of a successful nanoparticle conjugation.

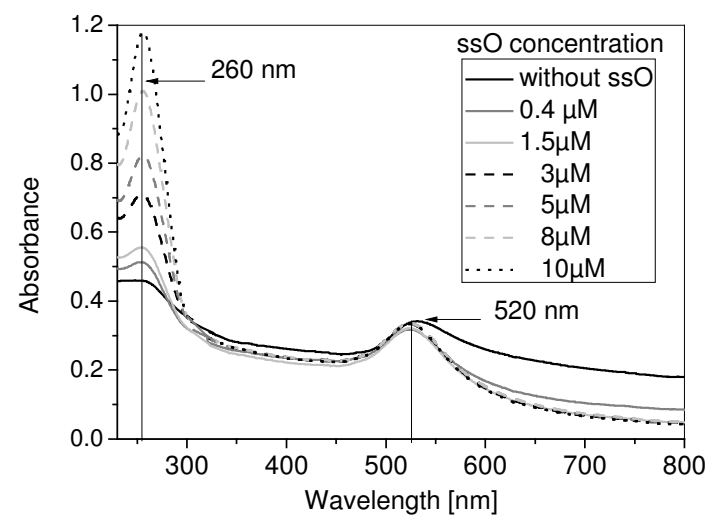

Figure 5 UV-VIS spectra of bioconjugates with different oligonucleotide concentrations 


\section{Conclusion}

We have established a novel single step approach to pure nanoparticle-bioconjugates without limitations in the variety of combinations between core material and organic shell. The in-situ conjugation during ultrashort pulsed laser ablation in liquids allows both, the generation of nanoparticles and their (bio-)conjugation. The mechanism of the method is based on fundamental electron donor (biomolecule) - electron acceptor (nanoparticle) attraction, so that a huge variety of materials bearing these basic properties are conjugable in future. We report quenching of nanoparticle growth and modification of the surface plasmon resonance as evidence of a successful functionalisation. The stabilization of gold nanoparticle in hexane using DDT and the first in-situ bioconjugation with ssO in aqueous media was achieved using the novel approach.

In summary, the in-situ conjugation of nanoparticles during laser ablation in liquids presents a novel route to the development of nanomaterials with added value for biomedical application.

\section{Acknowledgment}

The work was funded by the German Research Foundation within the excellence cluster REBIRTH. The authors like to acknowledge R. Gebauer for the registering of SEM micrographs.

\section{References}

1 Jordan, A., Scholz, R., Wust, P., Schirra, H., Schiestel, T.; Schmidt, H.; Felix, R J. Magn. Magn. Mater. 1999, 194, 185-196.

2 Gao, X.; Cui, Y.; Levenson, R.M.; Chung, L.W.K.; Nie, S. Nat. Biotechnol. 2004, 22, 969-976.

3 Rosi, N.L.; Giljohann, D.A.; Thaxton, C.S.; Lytton-Jean, A.K.R.; Han, M.S.; Mirkin, C.A. Science 2006, 312, 10271030 .

[4] Chen, J.; Saeki, F.; Wiley, B.J.; Chang, H.; Cobb, M.J.; Li, Z.Y.; Au, L.; Zhang, H.; Kimmey, M.B.; Li, X.; Xia, Y. Nano Lett. 2005, 5, 473-477.

[5] Han, M.S.; Lytton-Jean, A.K.R.; Oh, B.K. ; Heo, J. ; Mirkin, C.A. Angew. Chem. Int. Ed. 2006, 45, 1807-1810. [6] Anastas, P.T.; Warner, J.C. Green Chemistry: Theory and Practice (Oxford University Press, New York, 1998).

[7] Kabashin, A.V.; Meunier, M.; J. Appl. Phys. 2003, 94, 7941.

[8] Barcikowski, S.; Hahn, A.; Kabashin, A.V.; Chichkov, B.N. J. Appl. Phys. A. 2007, 87, 47-55

[9] Barcikowski, S., Menéndez-Manjón, A., Chichkov, B., Brikas, M., Račiukaitis, G. Appl. Phys. Lett. 2007, 91, 083113.

[10]Sylvestre, J.P.; Poulin, S.; Kabashin, A.V. ; Sacher, E. ; Meunier, M. ; Luong, J.H.T. J. Phys. Chem. B 2004, 108, 16864.

[11] Brust, M.; Bethell, D.; Schiffrin, D.J.; Kiely, C.J. Adv Mater. 1995,7,795.
[12] Compagnini, G.; Scalisi, A.A.; Puglisi, O. J. Mater. Res. 2004, 19, 2795-2798.

[13] Petersen,S.; Jakobi, J.; Barcikowski, S. Biomaterialien 2007, 8, 155-156.

[14]Dougan, J.A.; Karlsson, C.; Smith, W.E., Graham, D. Nucleic Acids Res. 2007, 35, 3668-3675.

[15]Y. Takeda, T. Kondow, F. Mafuné, J. Phys. Chem. B 2006, 110, 2393-2397.

[16] The $\mathrm{d}_{50}$ value is defined as particle diameter for $50 \%$ and collection efficiency in Hinds, W.C. Aerosol Technology - Properties, Behavior, and Measurement of Airborne Particles, 2nd ed.; Wiley-VCH: New York, 1998. [17]Jakobi, J.; Bärsch, N.; Petersen, S.; Hahn, A., Barcikowski, S. Proceedings of ICALEO 2007. [18]Hahn, A.; Barcikowski, S.; Chichkov, B.N. Proceedings of LPM 2007.

(Received: June 16, 2008, Accepted: March 12, 2009) 